\title{
STARLIB: A Next-Generation Reaction-Rate Library for Nuclear Astrophysics
}

\author{
A. L. Sallaska*, C. Iliadis, A. E. Chapmagne \\ University of North Carolina/TUNL \\ E-mail: sallaska@tunl.duke.edu
}

\section{F. X. Timmes, S. Starrfield}

Arizona State University

\section{S. Goriely}

Institut d'Astronomie et d'Astrophysique, Université Libre de Bruxelles, C.P. 226, B-1050

Brussels, Belgium

\begin{abstract}
None of the currently available stellar reaction-rate libraries include any information on reaction rate uncertainties. Although estimates have been attempted by some rate evaluations, their uncertainties are generally not based on rigorous statistical definitions. Clearly, a common standard for deriving uncertainties is warranted. STARLIB is a new, next-generation reaction-rate library that addresses this deficiency by providing a tabular, up-to-date database that supplies not only the recommended rate and its factor uncertainty but also the rate distribution (i.e. its probability density function PDF). The foundation of this library rests on an entirely new method for calculating reaction rates: Monte-Carlo simulation, which utilizes experimental nuclear physics quantities as inputs, yields a PDF for the reaction rate at a given temperature. From the cumulative distribution of rate probability densities, the low, median, and high rates are naturally defined. In addition, quantities with upper limits are seamlessly included. STARLIB bridges the gap between experimental nuclear physics data and stellar modelers by providing a convenient tabular format with reliable uncertainties and PDFs for use in the simulation of astrophysical phenomena. The library includes all types of reactions of astrophysical interest up to $Z=83$, such as $(p, \gamma),(p, \alpha),(\alpha, n)$, corresponding reverse rates, and weak interactions. Here, we introduce the library, summarize its construction, and offer a method for accessing it on the Internet.
\end{abstract}

XII International Symposium on Nuclei in the Cosmos

August 5-12, 2012

Cairns, Australia

\footnotetext{
* Speaker.
} 


\section{Motivation: Need for New Libraries}

Many reaction rate evaluations do not report uncertainties. Those that do, report "lower" and "upper" limits that have no rigorous, statistical definition (e.g., NACRE [1]). No uncertainties are included in current libraries (REACLIB [2], BRUSLIB [3]), which can lead to erroneous conclusions as some rates are uncertain by orders of magnitude. An important question also arises: when provided recommended, upper, lower rates, how does one interpolate among these values? In other words, how is the rate distributed? In order to model reaction rates realistically in Monte Carlo nucleosynthesis studies, the rate probability density function needs to be determined. STARLIB, a next-generation library whose structure is based on a Monte Carlo technique, connects stellar modelers with experimental data. Not only are reliable uncertainties included but also the approximate reaction rate probability density function. Here, we present the Monte Carlo method of calculating reaction rates, followed by a discussion of the library, its construction, and its website.

\section{Monte Carlo Based Rate Method}

The reaction rate, $N_{A}\langle\sigma v\rangle$, at a temperature $T$ for two interacting charged particles whose velocities are distributed according to Maxwell-Boltzmann is given by [4]:

$$
N_{A}\langle\sigma v\rangle=N_{A}\left(\frac{8}{\pi \mu}\right)^{1 / 2} \frac{1}{(k T)^{3 / 2}} \int_{0}^{\infty} E \sigma(E) e^{-E / k T} d E,
$$

where $N_{A}$ is Avogadro's number, $\mu$ is the reduced mass of the particles in the entrance channel, $E$ is the center-of-mass energy of the interaction, and $\sigma$ is the reaction cross section. A thorough description of the Monte Carlo method to calculate reaction rates can be found in the 2010 evaluation of Iliadis and collaborators [5] and in the forthcoming publication on the STARLIB library itself [6]. In brief, the Monte Carlo procedure assigns physically motivated distributions to each input parameter and randomly samples Eq. 2.1 until the desired precision set by the user is reached. A sample size of 5,000 is usually sufficient for the rate to vary by less than a few percent. The input distributions are Gaussian for resonance energies, lognormal for resonance strengths and nonresonant S-factors, Porter-Thomas for upper limits, and binary for interferences. The output of this calculation is the reaction rate probability density function, an example of which is shown in Fig. 1.

The top panel of Fig. 1 illustrates the rate output probability density function for a single, hypothetical narrow resonance in ${ }^{22} \mathrm{Na}(p, \gamma){ }^{23} \mathrm{Mg}$ at a fixed temperature for 10,000 samples $(\omega \gamma=$ $1.7 \pm 0.2 \mathrm{eV}$ at $\left.E_{r}=430 \pm 1 \mathrm{keV}\right)$. The lognormal approximation, shown as a black curve, is not a fit and is calculated directly from the output distribution. Details on lognormal functions are given in the following subsection. The cumulative probability distribution of the reaction rate is shown in the bottom panel of Fig. 1. From this distribution, the low rate (LR), recommended median rate (MR), and high rate (HR) are rigorously defined as the 16th, 50th, and 84th quantiles, respectively. A three-dimensional view of the top panel of Fig. 1 is shown in Fig. 2 for all temperatures.

\subsection{Lognormal Distributions}

A lognormal distribution is a distribution in which the logarithm of a variable $x$ is normally distributed and is defined by: 

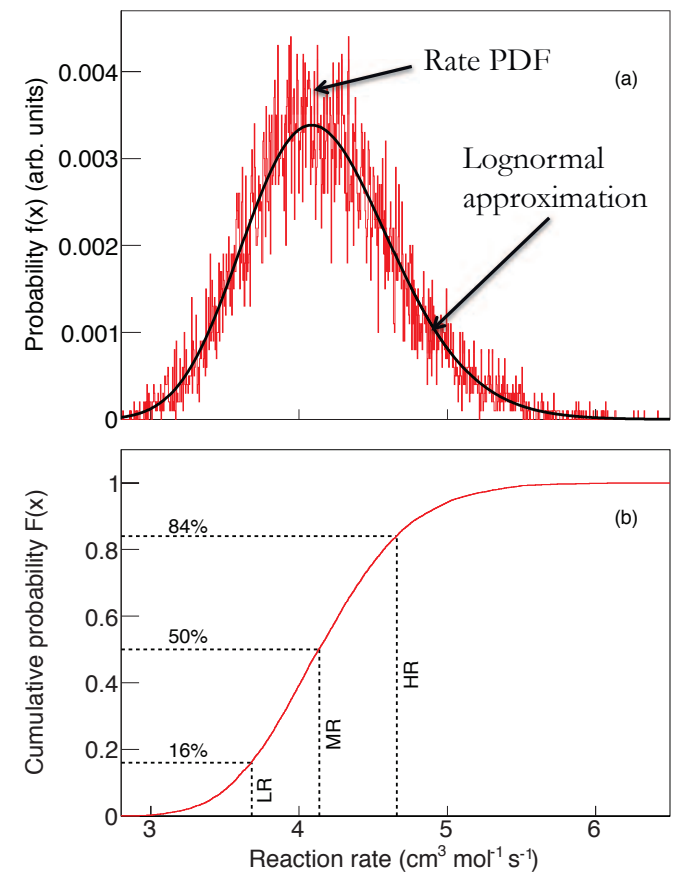

Figure 1: Sample Monte Carlo output for a hypothetical resonance (see text). Panel (a): reaction rate probability density. Red histogram: output of simulation. Black curve: lognormal rate approximation. Panel (b): cumulative probability distribution.

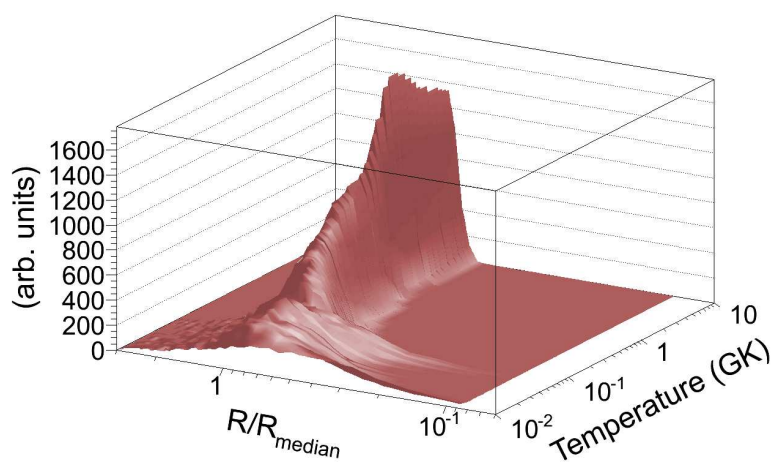

Figure 2: Three-dimensional reaction rate probability density function, normalized to the recommended rate. Notice how the distribution becomes narrower with increasing temperature, i.e., the reaction rate uncertainty decreases because the nuclear physics input becomes more reliable at higher energies. The top panel of Fig. 1 is a two dimensional view of this plot at one temperature.

$$
f(x)=\frac{1}{\sigma \sqrt{2 \pi}} \frac{1}{x} e^{-(\ln x-\mu)^{2} /\left(2 \sigma^{2}\right)} \quad \text { for } \quad 0<x<\infty .
$$

This function, similar to the Gaussian function, is parameterized by two constants, $\mu$ and $\sigma$ and is defined for only positive values of $x$. Its distribution is well-suited to describe distributions composed from multiplicative variables, such as resonance strengths. In contrast, distributions composed from additive variables, such as resonance energies, are more accurately characterized by Gaussian distributions. Although the lognormal distribution is asymmetric, in the case where the variance of the distribution is small when compared to the expectation value, the Gaussian distribution and lognormal distribution appear nearly indistinguishable.

The majority of reaction rates can be well-approximated with a lognormal distribution. Examples have been described in detail in Refs. [5, 6]. The rate output distribution is determined by the interplay of all input distributions and, thus, changes shape when one contribution dominates over another. If the rate is influenced most by a direct capture contribution, the resulting output PDF will be lognormal, as the $\mathrm{S}$-factor is given a lognormal distribution. This will also be true for single narrow resonances, regardless of which uncertainty dominates. A case where the output will not be lognormally distributed is one in which a contribution from a partial width upper limit dominates, as we assign a Porter-Thomas distribution to dimensionless reduced widths. 


\subsection{Modification of Laboratory Rates}

STARLIB is a stellar rate library. The majority of laboratory rates are measured with the target in its ground state; however, levels additional to the ground state are populated in stellar plasmas with a probability proportional to the Boltzmann factor. These levels will also contribute to the reaction rate. To convert the laboratory rates into stellar rates in STARLIB, we use stellar enhancement factors, $S E F$ s, calculated by the TALYS code [10]:

$$
N_{A}\langle\sigma v\rangle_{\text {stellar }}=S E F * N_{A}\langle\sigma v\rangle_{\text {lab }}
$$

In stellar plasmas at elevated temperature, reverse rates begin to play an important role. Reverse rates are calculated according to [4], with partition functions also calculated using the TALYS code [10].

\section{STARLIB Library}

STARLIB is a next-generation tabular, stellar thermonuclear reaction rate library based mainly on experimental data. In contrast to other libraries that offer only temperature and reaction and decay rates, STARLIB contains uncertainty information and probability density functions in order to facilitate more realistic simulations of energy generation and nucleosynthesis in various stellar phenomena. Because a majority of rates are well-approximated by lognormal distributions, this is accomplished by supplying only three quantities: temperature, rate $\left(x_{m e d}\right)$, and factor uncertainty (f.u.). Applying the properties discussed in Sec. 2.1, we find that for a reaction rate $x$ :

$$
x_{\text {med }}=e^{\mu}=\sqrt{x_{\text {high }} x_{\text {low }}}, \quad \text { f.u. }(68 \% \text { coverage })=e^{\sigma}=\sqrt{x_{\text {high }} / x_{\text {low }}} .
$$

The high and low rates (68\% coverage) are given by:

$$
x_{\text {low }}=e^{\mu} / e^{\sigma}=x_{\text {med }} / f . u ., \quad x_{\text {high }}=e^{\mu} * e^{\sigma}=x_{\text {med }} * f . u .
$$

Thus, by supplying the temperature, rate, and factor uncertainty, one can obtain not only the high rate $\left(x_{\text {high }}\right)$ and low rate $\left(x_{\text {low }}\right)$ but also the lognormal parameters $\mu$ and $\sigma$. With this information, the probability density function can be computed for any given temperature grid point.

\begin{tabular}{llll}
\hline \hline Rate & Number & Source & Uncertainties \\
\hline Monte Carlo (experimental) & 62 & Sallaska et al. [6]; Iliadis et al. [5] & individual \\
NACRE & 24 & Angulo et al. [1] & individual \\
Big Bang & 9 & Descouvemont et al. [7] & individual \\
$\beta$-decays (experimental) & 2225 & Audi et al. [8] & individual \\
$\beta$-decays (theoretical) & 5556 & Tuli, Nuclear Wallet Cards [9] & individual \\
TALYS (theory) & 47,721 & Goriely et al. [10] & factor of 10 \\
Neutron capture & 281 & Goriely et al. [10]; Xu et al. [3]; Bao et al. [11] & individual \\
${ }^{26}$ Al $\gamma$-ray transitions & 17 & Iliadis et al. [12] & individual \\
\hline \hline
\end{tabular}

Table 1: Summary of STARLIB rate sources and assumptions for uncertainties. Forward rates only. 
A list of the sources for STARLIB is shown in Table 1. First and foremost, STARLIB is an experimental library, and Monte Carlo based rates are paramount, if they are available. Updates to Monte Carlo rates since the evaluation of Ref. [5] include ${ }^{18} \mathrm{O}(p, \gamma){ }^{19} \mathrm{~F},{ }^{18} \mathrm{O}(p, \alpha){ }^{15} \mathrm{~N}$, ${ }^{22} \mathrm{Ne}(p, \gamma){ }^{23} \mathrm{Na},{ }^{22} \mathrm{Ne}(\alpha, \gamma){ }^{26} \mathrm{Mg},{ }^{22} \mathrm{Ne}(\alpha, n){ }^{25} \mathrm{Mg},{ }^{22} \mathrm{Na}(p, \gamma){ }^{23} \mathrm{Mg}$, and ${ }^{29} \mathrm{P}(p, \gamma){ }^{30} \mathrm{~S}$. However, experimental data exists for many rates that have not been computed with our Monte Carlo technique. For those rates, evaluations such as NACRE [1] and Descouvemont [7] supplement our library. Also included are both experimental $(\sim 550)$ and theoretical $\beta$ decays $(\sim 1700)$, as well as special links for reactions involving the ground and isomeric states of ${ }^{26} \mathrm{Al}$. Single-temperature experimental neutron capture rates [11] are extrapolated to other temperatures by renormalizing TALYS rates $[3,10]$.

For a plethora of reactions, nuclear properties have not been measured. The TALYS code [10] was used to estimate reaction rates in these cases. TALYS was also used to extrapolate neutron capture rates $[3,11]$ from the experimentally determined rate at a single energy to all STARLIB temperatures. For rates not available from the above sources, JINA's REACLIB [2] rates were converted from the analytical fit into our format. Reaction network studies that have used STARLIB are discussed in Refs. [12, 13].

\subsection{Format}

STARLIB is a tabular reaction rate library, consisting of a header and three columns for each reaction. The header is a singe line, including the type of interaction (i.e., the chapter number of JINA REACLIB [2]), the nuclei involved, the source reference, and the Q-value. Following are three columns: temperature, rate, and factor uncertainty. The temperature (in GK) is on a grid of 60 values between $1 \mathrm{MK}$ and $10 \mathrm{GK}$, and the rate is either the thermonuclear rate in units of $\mathrm{cm}^{3} \mathrm{~mol}^{-1} \mathrm{~s}^{-1}$ or the decay constant of photodisintigration or $\beta$ decay in units of $\mathrm{s}^{-1}$. The factor uncertainty is the number which multiples and divides the rate to calculate the upper and lower rate limits, respectively (Eq. 3.2). To calculate the probability density function parameters, for a given temperature the logarithm of the second column returns the value of $\mu$, and $\sigma$ is computed as logarithm of the third column, as discussed in the previous subsection.

\section{STARLIB Website: starlib.physics.unc.edu}

For ease of distribution and updating the STARLIB library within the stellar modeling and nuclear data communities, we have built a website at starlib.physics.unc.edu (see Ref. [14]). The site consists of three main sections: Rate Library, Rate Calculator, and Details.

Within the Rate Library page, one may display an individual rate and download the library or a subset. To display a rate, three menu boxes are available, depending on the desired form of the input. Formatting options are listed, as well as a button which cycles through examples directly in the boxes. A subset of the library may be downloaded by copying and pasting a list of required nuclei in the text box. Archived versions of the library will be also be accessible.

The Rate Calculator page houses a repository for our Monte Carlo input files and allows the calculation of individual rates with our code on our server. This is useful if, for example, one would like to examine how newly measured nuclear data affects the rate. We recommend editing an existing input file, as the formatting is important (see Refs. [5, 6] for details). Once the file is copied 
and pasted into the text box, starting the simulation requires only a valid email address. A priority queue system is used, and, depending on the input, the simulation is filtered into an appropriate queue and issued a job ID. Simulations may be monitored and output files may be downloaded at any time by returning to the bottom of the main Rate Calculator page and entering the email address used to start the simulation. Monitoring parameters include job ID, status (running or waiting in the queue), run time, progress percentage, and estimated time remaining. Upon completion of the simulation, an alert email is sent to the address provided with a link to the main Rate Calculator page, described above.

The Details page outlines the construction of STARLIB, its formatting, and updates. All references and links to original papers are included.

\section{Summary}

We have developed a next-generation stellar reaction rate library called STARLIB. Its structure is based on Monte Carlo rate simulations with experimental nuclear data as inputs and includes not only statistically rigorous uncertainties but also realistic rate distributions for stellar modelers. We have constructed a website at starlib.physics.unc.edu for ease of distribution within the community.

\section{Acknowledgments}

This work was supported in part by the National Science Foundation under award number AST-1008355 and by the Department of Energy under grant number DE-FG02-97ER41041.

\section{References}

[1] C. Angulo et al., Nucl. Phys. A 656, 3, (1999).

[2] R. H. Cyburt et al., Ap. J. Suppl. Ser., 189, 240 (2010); groups.nscl.msu.edu/jina/reaclib/db/index.php

[3] Y. Xu et al., Astron. and Astrophys. (submitted); www.astro.ulb.ac.be/bruslib

[4] C. Iliadis, Nuclear Physics of Stars (Weinheim: Wiley-VCH, 2007).

[5] R. Longland et al., Nucl. Phys. A 841, 1 (2010); C. Iliadis et al., Nucl. Phys. A 841, 31 (2010); C. Iliadis et al., Nucl. Phys. A 841, 251 (2010); C. Iliadis et al., Nucl. Phys. A 841, 323 (2010).

[6] A. L. Sallaska et al., Ap. J. Suppl. Ser. (to be submitted).

[7] P. Descouvemont et al., At. Data and Nucl. Data Tables, 88, 203 (2004).

[8] G. Audi et al., Nucl. Phys. A 729, 337 (2003); priv. commun. April 2011 by G. Audi and W. Meng.

[9] J. K. Tuli, Nuclear Wallet Cards, National Nuclear Data Center (2012).

[10] S. Goriely, S. Hilaire, A. J. Koning, Astron. and Astrophys., 487767 (2008).

[11] Z. Y. Bao et al., At. Data and Nucl. Data Tables, 76, 70 (2000).

[12] C. Iliadis, A. Champagne, A. Chieffi, and M. Limongi, Astrophys. J. Suppl. Ser. 193, 16 (2011).

[13] L. Downen et al., Ap. J. (submitted).

[14] A. L. Sallaska and C. Iliadis, Proceedings of Science for NIC XII. 